\title{
BEM-ESTAR SUBJETIVO EM PARTICIPANTES DO PROGRAMA MULHERES MIL, NA CIDADE DE BARBACENA - MG
}

\author{
F. Furtado', J. F. Antunes, L. M. M. Santos, L. A. Oliveira, R. S. Ferreira e B. M. M. Alves \\ Instituto Federal do Sudeste de Minas Gerais \\ fabianne.furtado@ifsudestemg.edu.br ${ }^{1}$
}

\section{RESUMO}

Introdução: as mulheres já são mais da metade da população brasileira. Entretanto, na hora da busca pelo emprego, o público feminino está em desvantagem. Muitas não tem qualificação e experiência profissional. O Programa Mulheres Mil foi implantado nos Institutos Federais (IFs) com o objetivo de oferecer as bases de uma política social de inclusão, por meio do qual as mulheres em situação de vulnerabilidade social tem acesso à educação profissional, emprego e renda. Objetivo: avaliar o bem-estar subjetivo em participantes do Programa Mulheres Mil, na cidade de Barbacena-MG. Métodos: Após aprovação do Comitê de Ética em Pesquisa, 41 mulheres de 5 bairros distintos da cidade de Barbacena foram selecionadas, de acordo com critérios de inclusão pré-determinados. No dia da matrícula, responderam, por meio de entrevista, ao Questionário de Saúde Geral e às escalas de Autoestima, de Ansiedade e Depressão, de Afetos Positivos e Negativos e de Satisfação com a Vida, todos cientificamente validados para uso no Brasil. Posteriormente, após 3 e 6 meses de participação no Programa, todas as mulheres foram reavaliadas. Resultados: nos 30 e 60 meses, as alunas apresentaram melhora nos itens que avaliaram a saúde geral. Apesar de estatisticamente não significante, clinicamente a satisfação com a vida progrediu favoravelmente durante a participação no Programa.

PALAVRAS-CHAVE: Mulheres, Vulnerabilidade, Bem-estar social, Autoestima, Afeto.

\section{SUBJECTIVE WELL-BEING OF PARTICIPANTS IN THE THOUSAND WOMEN PROGRAM IN THE CITY OF BARBACENA - MG}

\section{ABSTRACT}

Introduction: More than half of the Brazilian population are women. However, the female population is in disadvantage when they have to look for a job. Most of them do not have the necessary skills and professional experience. The Thousand Women Program was introduced in the Federal Institutes (IFs) to provide the basis for a social inclusion policy so that women in situation of social vulnerability could have access to professional education, employment and income. Objective: To assess the subjective well-being of the participants in the Thousand Women Program, in the city of Barbacena-MG. Methods: After being approved by the Research Ethics Committee, 41 women from 5 different neighborhoods of the city of Barbacena were selected, following the predetermined inclusion criteria. On the application day, the participants answered the General Health Questionnaire (GHQ-12), the Anxiety, Depression and Self-Esteem scales, the Positive and Negative Affect Scale, and the Satisfaction With Life Scale, which were all validated for use in Brazil. After 3 and 6 months, respectively, all women were reevaluated. Results: In 3 and 6 months, the students showed improvement in items that assessed their overall health. Although not statistically significant, clinically, satisfaction with life has increasingly improved during the participation in the program.

KEY-WORDS: Women, Vulnerability, Social Welfare, Self Concept, Affect. 


\section{BEM-ESTAR SUBJETIVO EM PARTICIPANTES DO PROGRAMA MULHERES MIL, NA CIDADE DE BARBACENA - MG}

\section{INTRODUÇÃO}

As mulheres já são mais da metade da população do país. De acordo com o Censo 2010, dos 190.755.799 residentes no país, 97.348.809 são mulheres. Apesar de as mulheres serem a maioria, a desigualdade de gênero ainda está presente no Brasil. Dados do IBGE mostram que $73 \%$ das esposas ganham menos que os maridos, apesar do aumento crescente da responsabilidade delas no comando da família. Entre 2001 e 2009, o número de mulheres chefes de família cresceu de $27 \%$ para $35 \%$ (INSTITUTO BRASILEIRO DE GEOGRAFIA E ESTATÍSTICA, 2010).

Na hora da busca pelo emprego, o público feminino também está em desvantagem. Muitas não tem qualificação e experiência profissional. Em 2007, dos mais de 9 milhões de trabalhadores à procura de emprego, apenas 1,6 milhão possuíam experiência e qualificação (SOUZA, 2007).

O Programa Mulheres Mil foi implantado com o objetivo de oferecer as bases de uma política social de inclusão, por meio do qual as mulheres tem acesso à educação profissional, emprego e renda. O programa Mulheres Mil está inserido no conjunto de prioridades das políticas públicas do Governo Federal, especialmente nos eixos promoção da eqüidade, igualdade entre sexos, combate à violência contra mulher e acesso à educação. Faz parte das ações do programa Brasil Sem Miséria. Os Institutos Federais de Educação, Ciência e Tecnologia (IFs), em conjunto com instituições parceiras, são os responsáveis pela formulação e execução dos projetos locais, que deverão estar de acordo com as necessidades da comunidade e com a vocação econômica regional, visando, simultaneamente, o aumento da escolaridade e a inserção do mercado formal.

No contexto internacional, o Programa também contribuiu para o alcance das Metas do Milênio promulgadas pela ONU em 2000 e aprovada por 191 países. Entre as metas estabelecidas estão a erradicação da pobreza extrema e da fome, promoção da igualdade entre os gêneros, autonomia das mulheres e garantia da sustentabilidade ambiental.

Os critérios utilizados para a escolha dos municípios foram: regiões de baixo índice de desenvolvimento humano (IDH), com semelhantes características econômicas e culturais e disponibilidade para instalação do escritório de acesso, onde as beneficiárias poderiam ser assistidas. A prioridade deveriam ser mulheres em situação de vulnerabilidade social.

A vulnerabilidade social pode ser entendida como um espelho das condições de bemestar social. Quando se fala em bem-estar social englobam-se dois sub-componentes: o bemestar econômico (salário/renda) e o bem-estar sócio-demográfico (moradia, saúde, alimentação, educação, lazer e outros). Todavia, cada pessoa pode reagir diferentemente a situações semelhantes. As experiências são percebidas e construídas socialmente através de crenças, pressuposições e expectativas sobre o mundo (ALBUQUERQUE; TRÓCCOLI, 2004). A avaliação que as pessoas fazem de suas vidas caracteriza o Bem-Estar Subjetivo (BES). Reflete a percepção 
e avaliação que o indivíduo faz de sua própria vida em termos de seus estados afetivos, funcionamento psicológico e social.

Pereira (1997) escreveu sobre os principais indicadores do bem-estar subjetivo, que são: aspecto cognitivo, aspecto afetivo e aspectos psicossociais da saúde mental. O primeiro está relacionado a uma experiência cognitiva em que o indivíduo compara sua situação atual com aquela que ele esperava estar, ou seja, o sucesso que a pessoa crê ter alcançado frente a algum objetivo desejado. $O$ segundo é a experiência dos aspectos afetivos, que pode ser medida através da freqüência de episódios positivos e negativos ocorridos num passado recente. $O$ terceiro se refere a fatores psicossociais da saúde mental, que podem incluir elementos como o próprio locus de controle, ansiedade, depressão, otimismo, autoestima, estresse, etc.

BES elevado inclui freqüentes experiências emocionais positivas, rara experiência emocional negativa (depressão ou ansiedade) e satisfação não só com vários aspectos da vida, mas com a vida como um todo. Naturalmente que o humor das pessoas, suas emoções e julgamentos valorativos, mudam com a passagem do tempo, caracterizando a satisfação com a vida como um construto não só multidimensional, mas também dinâmico.

A participação no Programa poderá constituir-se em fator de mudança na vida dessas mulheres por favorecer a inclusão social, por meio da oferta de formação focada na autonomia e na criação de alternativas para a inserção no mundo do trabalho. Dessa maneira, poderá influenciar na satisfação com a vida, felicidade, afeto positivo e negativo - componentes do BES das participantes.

\section{OBETIVO}

Avaliar o Bem-Estar Subjetivo em participantes do Programa Mulheres Mil, na cidade de Barbacena.

\section{MÉTODOS}

O presente estudo é do tipo clínico, primário, analítico, prospectivo e longitudinal. As participantes deram o seu aval por meio da assinatura do Termo de Consentimento Livre e Esclarecido (TCLE).

Casuística

Critérios de inclusão

Das 100 mulheres selecionadas pela Coordenação do Programa Mulheres Mil, nos bairros Nove de Março, Santa Maria, Ponto Chic do Martelo, São Francisco e Nova Cidade da cidade de Barbacena (MG), foram incluídas 66 mulheres, de maneira consecutiva, as quais se enquadraram nos critérios estipulados pelo Ministério da Educação (MEC) para inclusão no Programa:

- Idade mínima de 18 anos;

- Baixo nível de alfabetização;

- Dificuldade de aprendizagem;

- Pobreza acentuada;

- Condições de moradia precária;

- Baixa autoestima; 
- Histórico de emprego com baixa remuneração e condições adversas;

- Necessidade de acesso aos serviços públicos;

- Fragilidade da estrutura de apoio familiar;

- Estado de vulnerabilidade social;

Critério de Exclusão

Foram excluídas as voluntárias que não completaram qualquer uma das etapas do estudo.

Delineamento do Estudo

O presente estudo consistiu de três etapas: (1) a Etapa Preliminar (EP), antes da realização de qualquer atividade do Programa pelas participantes; (2) Etapa Intermediária (EI), no 3o mês, a partir do início do Programa; (3) Etapa Final (EF), imediatamente após o término do Programa, o que ocorreu seis meses após o início. Em cada uma das etapas as participantes responderam às escalas e ao questionário abaixo descritos.

Durante os meses de participação no Programa foram desenvolvidas atividades: para elevação da autoestima; de profissionalização; e para elevação da escolaridade (somente para aquelas que não possuíam o Ensino Médio Completo).

Instrumentos

Foram aplicados em um ambiente claro e silencioso, por meio de entrevista, na sequência: saúde geral, autoestima, ansiedade e depressão, afetos positivos e negativos e satisfação com a vida.

\section{Questionário de Saúde Geral (GSQ-12)}

Este instrumento corresponde a uma versão abreviada do Questionário de Saúde Geral de Goldberg (1972), adaptado para o Brasil por Pasquali et al. (1994). Como sua abreviação indica, a versão que é utilizada neste estudo se compõe de 12 itens (por exemplo: Você tem se sentido pouco feliz e deprimido?; Você tem perdido a confiança em si mesmo?). Cada item é respondido em termos do quanto a pessoa tem experimentado os sintomas descritos, sendo suas respostas dadas em uma escala de quatro pontos. No caso dos itens que negam a saúde mental (por exemplo: Suas preocupações the têm feito perder muito sono?; Tem se sentido pouco feliz e deprimido?), as alternativas de resposta variam de 1 (Absolutamente, não) a 4 (Muito mais que de costume); no caso dos itens afirmativos (por exemplo: Tem se sentido capaz de tomar decisões?; Tem podido concentrar-se bem no que faz?), as respostas foram de 1 (Mais que de costume) a 4 (Muito menos que de costume). Quanto menor a pontuação, melhor o nível de saúde mental.

\section{Escala de Autoestima de Rosenberg}

A Escala de Autoestima de Rosenberg (ROSENBERG, 1965), em sua versão traduzida e adaptada para o português (DINI, QUARESMA, FERREIRA, 2004) é composta de dez afirmativas com quatro opções de respostas que variam de nada importante a extremamente importante 
(concordo fortemente; concordo; discordo; discordo fortemente). Cada alternativa tem um valor que varia de zero a três. Quanto maior o escore, menor a autoestima do indivíduo. O escore final da escala pode variar de zero (melhor autoestima) a trinta (pior autoestima).

\section{Escala Hospitalar de Ansiedade e Depressão}

A Escala Hospitalar de Ansiedade e Depressão (The Hospital Anxiety and Depression Scale HADS) (ZIGMOND; SNAITH, 1983) tem 14 itens: 7 voltados para a avaliação da ansiedade (HADSA) e 7, para a depressão (HADS-D). No Brasil, foi validada por BOTEGA et al. (1998). Cada um dos seus itens pode ser pontuado de zero a três, compondo uma pontuação máxima de 21 pontos para cada subescala (ansiedade e depressão).

\section{Escala de Afetos Positivos e Negativos}

Esta medida foi originalmente proposta por Diener e Emmons (1984, conforme citado por Reis, Gable, Roscoe \& Ryan, 2000) para avaliar a valência dos afetos, tendo sido realizados estudos recentes que comprovam a adequação dos seus parâmetros psicométricos (OMODEl; WEARING, 1990). Esta escala se compõe de nove adjetivos, sendo quatro positivos e cinco negativos. Os afetos positivos são: feliz, alegre, satisfeito e divertido; e os negativos são: deprimido, preocupado, frustrado, raivoso e infeliz. Procurando equilibrar o número de adjetivos para ambos tipos de afetos, foi acrescentado nesta pesquisa um adjetivo para o afeto positivo: otimista. Este instrumento avalia o quanto o participante tem experimentado cada uma destas emoções nos últimos dias. Os itens são respondidos em uma escala de sete pontos, com os extremos 1 (Nada) e 7 (Extremamente).

\section{Escala de Satisfação com a Vida}

Esta medida foi proposta originalmente por Ed Diener, tendo sido realizados estudos recentes que comprovam a adequação dos seus parâmetros psicométricos. Por exemplo, Pavot; Diener (1993) descrevem uma consistência interna, Alfa de Cronbach, de 0,87; sua estabilidade temporal, considerando um período de dois meses, foi de 0,82 . Estes autores realizaram também uma análise fatorial com os dados obtidos, tendo observado um único fator que explicou $66 \%$ da variância total. Compõe-se de cinco itens, como os que seguem: estou satisfeito com minha vida e as condições da minha vida são excelentes, os quais são respondidos em uma escala tipo Likert, variando de 1 (Discordo Totalmente) a 7 (Concordo Totalmente).

Além dessas variáveis, um conjunto de perguntas de natureza sóciodemográfica foi inserido com o fim de caracterizar a casuística (por exemplo, idade, escolaridade, religião, dentre outras).

Os dados foram agrupados pelo programa Statistical Package of Social of Science (SPSS), versão 17.0. O teste de Friedman foi utilizado para comparação das pontuações dos instrumentos ente três as etapas. Foi adotado nível de significância de 5\%.

\section{RESULTADOS}

Quarenta e uma mulheres completaram todas as etapas do estudo. As características sóciodemográficas estão apresentadas na Tabela 1. A média de idade entre as alunas foi de 33,36 
anos; a maior parte era solteira e a porcentagem de casadas e amasiadas foi idêntica. Em relação à escolaridade, predominou a incompletude do ensino fundamental. Mais da metade das mulheres não possuíam renda própria (se ocupavam com os afazeres domésticos ou estavam desempregadas).

Tabela 1: Características sóciodemográficas.

\begin{tabular}{|c|c|c|c|}
\hline Variáveis & Média [desvio-padrão] & $\mathrm{N}$ (casuística) & $\%$ \\
\hline Idade (anos) & $33,36[10,76]$ & 41 & \\
\hline \multicolumn{4}{|l|}{ Estado Civil } \\
\hline Solteiro & & 16 & 39,03 \\
\hline Casada & & 12 & 29,27 \\
\hline Viúva & & 0 & 0 \\
\hline Divorciada & & 1 & 2,43 \\
\hline Amasiada & & 12 & 29,27 \\
\hline
\end{tabular}

\section{Escolaridade}

Estado fundamental completo

Estado fundamental incompleto

56,10

Estado médio completo

21,95

Estado médio incompleto

12,20

\section{Religião}

Católica

38

92,68

Evangélica

Espírita

0

0

Nenhuma

0

0

Profissão

Do lar/desempregada

Outras

Número de membros da família que

\section{moram na mesma casa}

$\begin{array}{lrr}1 \text { pessoa } & 1 & 2,43 \\ 2 \text { pessoas } & 12 & 29,27 \\ 3 \text { pessoas } & 6 & 14,65 \\ 4 \text { pessoas } & 14 & 34,14 \\ 5 \text { ou mais } & 8 & 19,51\end{array}$

Renda familiar

Até 1 salário mínimo

No 3ㅇ e 60 meses, as mulheres apresentaram melhora na Saúde Geral quando comparado ao momento inicial, antes do início da participação no Programa Mulheres Mil. Apesar de estatisticamente não significante, clinicamente a satisfação com a vida progrediu favoravelmente durante a participação no Programa. 
Tabela 2: Resultados dos questionários e escalas de avaliação do bem-estar subjetivo.

\begin{tabular}{|c|c|c|c|c|c|c|}
\hline Questionário e Escala & $\begin{array}{l}\text { Períodos de } \\
\text { avaliação }\end{array}$ & $\begin{array}{l}\text { Média } \\
(\mathrm{N}=41)\end{array}$ & $\begin{array}{c}\text { Mediana } \\
(\mathrm{N}=41)\end{array}$ & $\begin{array}{l}\text { Desvio- } \\
\text { padrão } \\
(\mathrm{N}=41) \\
\end{array}$ & & $p$-valor ${ }^{\ddagger}$ \\
\hline \multirow{3}{*}{$\begin{array}{c}\text { QSG-12 } \\
\text { (12-48 pontos) }\end{array}$} & EP (início) & 22.93 & 22 & 6.24 & \multirow{3}{*}{$0,002 *$} & Pré × 3 meses: $<0,05$ \\
\hline & El (3 meses) & 19.49 & 19 & 5.13 & & Pré X 6 meses: $<0,05$ \\
\hline & EF (6 meses) & 19.85 & 19 & 5.93 & & $3 \times 6$ meses: $\mathrm{ns}$ \\
\hline \multirow{3}{*}{$\begin{array}{c}\text { Escala de Autoestima } \\
\text { Rosenberg } \\
\text { (0-30 pontos) }\end{array}$} & EP & 10.00 & 10 & 4.87 & \multirow{3}{*}{0,37} & \\
\hline & $\mathrm{EI}$ & 9.66 & 10 & 4.41 & & \\
\hline & $\mathrm{EF}$ & 9.54 & 10 & 3.78 & & \\
\hline \multirow{3}{*}{$\begin{array}{l}\text { HADS - Ansiedade } \\
\text { (0-21 pontos) }\end{array}$} & EP & 7.76 & 7 & 3.62 & \multirow{3}{*}{0,84} & \\
\hline & $\mathrm{EI}$ & 7.15 & 7 & 3.42 & & \\
\hline & $\mathrm{EF}$ & 7.37 & 6 & 4.13 & & \\
\hline \multirow{3}{*}{$\begin{array}{l}\text { HADS - Depressão } \\
\text { (0-21 pontos) }\end{array}$} & EP & 5.41 & 5 & 2.89 & \multirow{3}{*}{0,06} & \\
\hline & $\mathrm{EI}$ & 4.05 & 4 & 3.02 & & \\
\hline & $\mathrm{EF}$ & 4.34 & 4 & 2.62 & & \\
\hline \multirow{3}{*}{$\begin{array}{l}\text { Afetos Positivos } \\
\text { (5-35 pontos) }\end{array}$} & EP & 23.93 & 25 & 4.67 & \multirow{3}{*}{0,40} & \\
\hline & $\mathrm{EI}$ & 24.32 & 26 & 5.91 & & \\
\hline & $\mathrm{EF}$ & 24.88 & 26 & 5.31 & & \\
\hline \multirow{3}{*}{$\begin{array}{l}\text { Afetos Negativos } \\
\text { (5-35 pontos) }\end{array}$} & EP & 15.41 & 14 & 5.55 & \multirow{3}{*}{0,17} & \\
\hline & $\mathrm{El}$ & 13.95 & 13 & 4.72 & & \\
\hline & $\mathrm{EF}$ & 14.44 & 13 & 6.69 & & \\
\hline \multirow{3}{*}{$\begin{array}{l}\text { Satisfação com a Vida } \\
\text { (5-35 pontos) }\end{array}$} & EP & 25.10 & 25 & 4.30 & \multirow{3}{*}{0,05} & \\
\hline & $\mathrm{EI}$ & 26.59 & 25 & 5.93 & & \\
\hline & $\mathrm{EF}$ & 26.88 & 25 & 4.26 & & \\
\hline
\end{tabular}

QSG: Questionário de Saúde Geral; HADS: Hospitalar Anxiety and Depression Scale; N: tamanho da

casuística; EP: Etapa preliminar (antes do início das atividades do Programa); El: Etapa intermediária (3o mês); EF: Etapa final (6o mês); ${ }^{\ddagger}$ Teste de Friedman; ns: não-significante; ${ }^{*} p<0,05$.

\section{DISCUSSÃO}

Tem-se presenciado nas últimas décadas inúmeras transformações na estrutura mundial. O modelo econômico vigente - capitalista - é marcado pela competição, busca de status, reconhecimento, supervalorização do self (individualismo) e consumismo (SILVA et al., 2009). Quem detém o capital é quem tem as melhores condições de moradia, acesso aos recursos, educação, etc. Enquanto isso, quem está do outro lado, os trabalhadores, que não detêm a renda nem o capital, estão na extremidade inferior da relação. Logo, percebe-se um contexto de desigualdade social, gerada primordialmente pela diferenciação econômica. No Brasil, a enorme desigualdade na distribuição de renda e os elevados níveis de pobreza exclui parte significativa da população do acesso a condições mínimas de dignidade e cidadania (GOMES \& PEREIRA, 2005). Cabe destacar que um país tem pobreza quando existe escassez de recursos ou quando, apesar de haver um volume aceitável de riquezas, elas estão mal distribuídas. O Brasil não é um país pobre, e sim um país desigual (GOMES \& PEREIRA, 2005). 
Em se tratando de exclusão, as mulheres, apesar de terem aumentado a participação no mercado de trabalho e melhorado o grau de instrução, continuam encabeçando a lista. Elas representam $55 \%$ da população mundial, mas, no entanto, apenas $40 \%$ estão no mercado de trabalho e representam apenas $10 \%$ da renda do mundo. A pobreza no mundo tem, portanto, sexo (SANTOS, 2012).

O termo exclusão social tem sentido temporal e espacial: um grupo social está excluído segundo determinado espaço geográfico ou em relação à estrutura e conjuntura econômica e social do país a que pertence. No Brasil, esse termo está relacionado principalmente à situação de pobreza, uma vez que as pessoas nessa condição constituem grupos em exclusão social, porque se encontram em risco pessoal e social, ou seja, excluídas das políticas sociais básicas (trabalho, educação, saúde, habitação, alimentação) (GOMES \& PEREIRA, 2005). Assim, à medida que o indivíduo encontra dificuldades para cumprir satisfatoriamente suas tarefas básicas de socialização e de amparo/serviços, criam-se situações de vulnerabilidade (PETRINI, 2003).

As freqüentes situações de instabilidade que gera conflitos, ansiedade, angústia e desestabilização emocional. $O$ aumento e a diversificação de papéis, divergências de situações, questões familiares e afetivas, bem como as questões de empregabilidade e desemprego, requerem cada vez mais ajustes que demandam energia, tanto físicas quanto psíquicas. Assim, é travada uma luta diária que perpassa pelas questões de sobrevivência e vai até os fatores relacionados à qualidade de vida e bem-estar (SILVA et al., 2009).

É neste cenário que cada vez mais é exigida do indivíduo uma adequação no desempenho de papéis, de forma que a variedade de compromissos do mundo moderno não o leve à sobrecarga e ao adoecimento. O equilíbrio tão desejado e ao mesmo tempo tão distante é visivelmente percebido pelas crescentes queixas cotidianas de sobrecarga, ansiedade patológica, sentimentos de impotência e depressão.

Segundo Diener \& Lucas (2000), o bem-estar subjetivo se refere ao que as pessoas pensam e como elas se sentem sobre suas vidas. Desse modo, pode-se entender que o bem-estar é composto por dois componentes: o cognitivo, chamado satisfação com a vida; e o afetivo. 0 primeiro diz respeito aos aspectos racionais e intelectuais, por exemplo, quando a pessoa faz um julgamento avaliativo consciente sobre a sua vida como um todo, ou de áreas específicas, como o lazer ou o trabalho; o segundo envolve os componentes emocionais, isto é, a adição dos humores e emoções, por exemplo, quando a pessoa experimenta emoções prazerosas ou desprazerosas. 0 aspecto cognitivo pode ser avaliado pela satisfação com a vida e/ou domínios específicos da vida, moral e qualidade de vida. $\mathrm{O}$ aspecto emocional pode ser avaliado pelo otimismo, felicidade, afeto positivo, afeto negativo, equilíbrio afetivo, depressão, ansiedade e distress (NOVO, 2003).

Os níveis de bem-estar subjetivo modificam-se quando eventos de vida recentes (positivos ou adversos) se desviam dos padrões normais, exigindo do indivíduo uma resposta adequada. No presente estudo, a melhora dos níveis de saúde mental pode ter sido alcançada em virtude da participação das atividades planejadas durante o Programa: aulas sobre Saúde da Mulher, Noções de Informática, Produção de Sabão, Etiqueta à mesa, Psicologia, Prevenção de Acidente Doméstico e Noções de Primeiros Socorros, Direito da Mulher, Saúde da Família e Saúde Bucal; Cursos de Serviços Gerais, Artesanato e Curso de Auxiliar de Cozinha; e aulas para certificação de escolaridade.

Apesar de os demais instrumentos utilizados serem pertinentes para o estudo do bemestar subjetivo, não houve diferença na pontuação entre as etapas inicial, intermediária e final. 
Talvez, a forma de estruturação e a linguagem dos instrumentos possam ter influenciado nesse resultado. Cabe destacar que apesar de a pontuação na Escala de Satisfação com a Vida não ter mostrado diferença estatística $(p=0,05)$, clinicamente esse dado se mostrou relevante.

O Questionário de Saúde Geral (QSG-12) permite avaliar a severidade de distúrbios psiquiátricos não-psicóticos e servir como meio de identificação de casos potenciais dos mesmos, na população geral não clínica. A escolha do QSG mostrou-se adequada para atender o objetivo proposto uma vez que ao considerar os fatores estresse, desejo de morte, desconfiança da capacidade de desempenho, distúrbios do sono, distúrbios psicossomáticos e saúde geral como indicadores para avaliar a qualidade de vida, consegue expressar itens comportamentais, enfatizar a severidade da ausência de saúde mental e expressar os sentimentos, tópicos de especial interesse em uma população sob elevada condição de risco, como a do presente estudo.

Este é o primeiro artigo publicado em revista científica com resultados do Programa Mulheres Mil. A associação entre Ensino, Pesquisa e Extensão apresenta-se, no âmbito das instituições de ensino como uma das maiores virtudes do compromisso social (MARTINS). Esperase que outros futuros possam ser apresentados à comunidade científica tanto para efeitos de cunho acadêmico quanto para o próprio aperfeiçoamento do Programa.

\section{CONCLUSÃO}

As alunas participantes da primeira turma do Programa Mulheres Mil na cidade de Barbacena - MG melhoram os níveis de Saúde Geral e Satisfação com a Vida.

\section{REFERÊNCIAS BIBLIOGRÁFICAS}

1. AlBUQUerque A.S., TRÓcColi, B.T. Desenvolvimento de Uma Escala de Bem-Estar Subjetivo. Psicologia: Teoria e Pesquisa, v.20, n.2, p. 143-164, maio./ago. 2004.

2. BOTEGA, N.J., PONDE, M.P., MEDEIROS P., LIMA, M.G., GUERREIRO, C.A.M. Validação da escala hospitalar de ansiedade e depressão (HAD) em pacientes epiléticos ambulatoriais. Jornal Brasileiro de Psiquiatria, n.47, v.6, p.285-289, jun. 1998.

3. DIENER, E.; LUCAS, R.E. Explaining differences in societal levels of happiness: Relative standards, need fulfillment, culture, and evaluation theory. Journal of Personality Assessement, v. 1, n. 1, p. 41-78, 2000.

4. DINI, G.M., QUARESMA, M.R., FERREIRA, L.M. Adaptação Cultural e Validação da Versão Brasileira da Escala de Autoestima de Rosenberg. Revista Brasileira de Cirurgia Plástica, v.19, n.1, p. 41-52, jan./abr. 2004.

5. GOLDBERG, D. The detection of psychiatric illness by questionnaire. London: Oxford University Press, 1972. $156 \mathrm{p}$.

6. GOMES, M.A., PEREIRA, M.L.D. Família em situação de vulnerabilidade social: uma questão de políticas públicas. Ciência \& Saúde Coletiva, v.10, n. 2, p. 357-363, 2005.

7. INSTITUTO BRASILEIRO DE GEOGRAFIA E ESTATíSTICA (IBGE). Sinopse do Censo Demográfico 2010: Tabela 1.11, População residente, por situação do domicílio e sexo, segundo as Grandes Regiões e as Unidades da Federação. In Rio de Janeiro, 2010. Disponível em: <http://www.ibge.gov.br/home/estatistica/populacao/censo2010/sinopse.pdf>. Acesso em: 22 out. 2011. 
8. MARTINS, M.L. Ensino-pesquisa-extensão como fundamento metodológico na construção do conhecimento na universidade. Disponível em: <http://www.ppg.ufrn.br/conteudo/documentos/cursoiniciacao/ensino_pesquisa_extensao.pdf> Acesso em: 17 jul. 2012.

9. NOVO, R.F. Bem-estar subjetivo e bem-estar psicológico: diferentes faces da eudaimonia. In (Org.), Para além da eudaimonia. O bem-estar psicológico em mulheres na idade avançada. Coimbra: Fundação Calouste Gulkenkian, 2003. p. 21-75.

10. OMODEI, M.M., WEARING, A.J. Need satisfaction and involvement in personal projects: toward an integrative model of subjective well-being. Journal of Personality and Social Psychology, v.59, p.762-769, 1990.

11. PASQUALI, L., GOUVEIA, V.V., ANDRIOLA, W.B., MIRANDA, F.J., RAMOS, A.L.M. Questionário de Saúde Geral de Goldberg (QSG): adaptação brasileira. Psicologia Teoria \& Pesquisa, v.10, p. 421437, 1994.

12. PAVOT, W., DIENER, E. Review of the satisfaction with life scale. Psychological Assessment, v.5, p. 164-172, 1993.

13. PEREIRA, C.A.A. Um panorama histórico-conceitual acerca das subdimensões da qualidade de vida e do bem-estar subjetivo. Arquivos Brasileiros de Psicologia, v.49, n.4, p. 32-48, 1997.

14. PETRINI, J.C. Pós-modernidade e família. Bauru: Edusc, 2003. 230 p.

15. REIS, H.T., SHELDON, K.M., GABLE, S.L., ROSCOE, J., RYAN, R.M. Daily well-being: the role of autonomy, competence, and relatedness. Personality and Social Psychology Bulletin, v.26, p. 419$435,2000$.

16. ROSENBERG, M. Society and the adolescent self image. Princeton: Princeton University Press; 1965. $347 \mathrm{p}$.

17. SANTOS, M.A. Desigualdade entre homens e mulheres persiste. A verdade, 1 mar. 2012. Disponível em: <http://averdade.org.br/2012/03/desigualdade-entre-homens-e-mulherespersiste>. Acesso em 17 jul. 2012.

18. SHELDON, K.M., KASSER, T. Coherence and congruence: Two aspects of personality integration. Journal of Personality and Social Psychology, v.68, p.531-543, 1995.

19. SILVA, D., PAZ, A.J.O., SOLIVA, B., COMIRAN, F., PICINI, G., SOUZA, L. B., SOTORIVA, M., SAVARIS, R.; MARÇAL, S.A.S. Bem-estar psicológico versus distress: um estudo exploratório sobre saúde mental. Akrópólis, v.17, n.2, p. 59-68, abr./maio 2009.

20. SOUZA, J.L. Trabalho - O mapa do emprego no Brasil. Desafios do Desenvolvimento, v.38, p. 64-65, dez. $2007 . \quad$ Disponível em: <http://desafios2.ipea.gov.br/sites/000/17/edicoes/38/pdfs/rd38sec03.pdf>. Acesso em: 22 out. 2011.

21. ZIGMOND, A.S., SNAITH, R.P. The hospital anxiety and depression scale. Acta Psychiatrica Scandinavica, v.67, n.6, p. 361-370, jun. 1983.

Agradecimentos

A todos da equipe: Romualdo Santarosa de Sousa, Viviane Caldeira Resende, Adriana Magalhães Veiga de Broutelles, Claudilene Márcia Figueiredo Ferrão, Bianca Alvin de Andrade Silveira, Eliane Loschi da Silva, Ênio Saviatto Falzoni Júnior, José Saraiva Cruz, Renata Cristina Condé, Maria Aparecida Garcia Pinheiro Goulart e Lívia Lanne Fávero. 